\title{
Metabolic syndrome and its associated early-life factors in children and adolescents: a cross-sectional study in Guangzhou, China
}

\author{
Jiao Wang ${ }^{1}$, Yanna Zhu ${ }^{1}$, Li Cai ${ }^{1}$, Jin Jing ${ }^{1}$, Yajun Chen ${ }^{1}$ * , Jincheng Mai ${ }^{2}$, Lu Ma ${ }^{1}$, \\ Yinghua $\mathrm{Ma}^{3}$ and Jun $\mathrm{Ma}^{3}$ \\ 'Department of Maternal and Child Health Care, School of Public Health, Sun Yat-Sen University, Guangzhou \\ 510080, People's Republic of China: ${ }^{2}$ Guangzhou Health Care Clinics of Middle and Primary Schools, Guangzhou, \\ People's Republic of China: ${ }^{3}$ Institute of Child and Adolescent Health, Public Health of Peking University, Beijing, \\ People's Republic of China
}

Submitted 9 April 2015: Final revision received 27 July 2015: Accepted 29 July 2015: First published online 8 September 2015

\begin{abstract}
Objective: The present study aimed to investigate the prevalence of metabolic syndrome (MetS) in 7- to 17-year-old children and adolescents in China and to examine the relationship between MetS and its associated early-life factors.

Design: Data were collected using a standard parent/guardian questionnaire in a face-to-face interview. Each participant underwent a complete anthropometric evaluation. MetS was defined according to the criteria of the International Diabetes Federation (IDF; 2007) for children and adolescents.

Setting: Guangzhou, a large city in South China, September 2013.

Subjects: A total of 1770 children and adolescents were enrolled in the study, including 913 girls (51.6\%) and 857 boys (48.4\%).

Results: The overall prevalence of MetS in children and adolescents was $1.1 \%$ ( $n$ 19), which was higher in boys (1.4\%) than in girls (0.8\%). Multivariate analysis indicated that high birth weight was significantly associated with abdominal obesity $(\mathrm{OR}=2 \cdot 86 ; 95 \%$ CI 1.62, 5.06) and MetS (OR $=3.61 ; 95 \%$ CI 1.33, 9.82). Furthermore, $>6$ months of maternal breast-feeding was inversely associated with MetS (OR $=0 \cdot 39$; $95 \%$ CI $0 \cdot 16,0 \cdot 98)$.

Conclusion: Based on IDF criteria, the prevalence of MetS among southern Chinese children was significantly lower than that in other populations. High birth weight was significantly associated with abdominal obesity and MetS, and breast-feeding for longer than 6 months was inversely associated with MetS in South China.
\end{abstract}

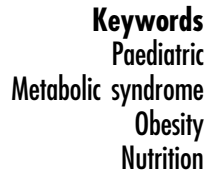

Metabolic syndrome (MetS) is a cluster of cardiovascular risk factors defined by the co-occurrence of at least three of the following features: obesity, hyperglycaemia, hypertension, hypertriacylglycerolaemia and low level of HDL cholesterol (HDL-C) ${ }^{(1)}$. The prevalence of MetS in children and adolescents is increasing worldwide. In 2014, Miller et al. reported that approximately $73.2 \%$ of the participants in their study presented at least one feature, with an estimated MetS prevalence of $10 \cdot 1 \%$ among adolescents in the USA ${ }^{(2)}$. A study in 2012 showed that the prevalence of MetS in six cities in China was $27.6 \%$ in obese participants and $0.2 \%$ in normal-weight children ${ }^{(3)}$.

Morrison et al. showed that MetS during childhood predicts adult MetS and type 2 diabetes mellitus 25-30 years later ${ }^{(4)}$. This finding emphasizes the importance of early control of MetS during childhood as a strategy for primary prevention of CVD later in life. Identification of risk factors in youngsters is crucial for the prevention and rapid detection of $\operatorname{MetS}^{(5)}$; however, the associated early-life factors of MetS in children remain unclear and previous results are inconsistent. Some studies showed that urbanization, unhealthy diet and sedentary lifestyle are major contributors to health disorders ${ }^{(6,7)}$. Furthermore, certain circumstances in utero and early childhood predispose a child to disorders such as obesity, dysglycaemia and $\operatorname{MetS}^{(8-10)}$. The association between childbirth and feeding situations and MetS is unclear; a strong association was reported between low birth weight (LBW) or high birth weight (HBW) and MetS in some studies, whereas this association was not found in other studies $^{(11,12)}$. Several reports also indicated that family environments such as maternal education level ${ }^{(13)}$ 
and family history of diabetes ${ }^{(14)}$ are risk factors for obesity and hyperinsulinaemia, which are the most important determinants of MetS. One study explored the prevalence of MetS and its associated factors in north-eastern Chinese adults ${ }^{(15)}$. However, only a few large studies have evaluated the relationship between early-life factors and MetS among children and adolescents in China.

Development of effective strategies and actions to control and prevent the prevalence of obesity-related chronic diseases is urgently needed in China. The present study aimed to provide an up-to-date prevalence of MetS in 7- to 17-year-old children in southern China and to examine the relationships of early predictors, such as birth weight, breast-feeding and parental education level, with MetS development. We tested the hypothesis that maternal breast-feeding and either LBW or HBW might be associated with MetS in Chinese children and adolescents.

\section{Method}

\section{Study population}

This cross-sectional study was conducted in September 2013 in Guangzhou, which is a large and wealthy city in South China. Based on multiple random sampling, four districts from the urban area were randomly selected in the first stage. In the second stage, 2108 eligible students aged $7-17$ years from ten schools in the four districts were invited to participate through cluster random sampling (primary school: secondary school =1:1). Among the ten selected schools, two classes per grade were randomly selected and invited, and all children aged 7-17 years were considered eligible in these classes. The principals (school heads) of selected schools were sent an invitation letter, an information sheet and a presentation containing the study details. With the permission from the principals, parent and student consent forms, including consents for physical examination and blood sample collection, were given to each student to bring home. The children were then asked to return the consent forms to the school if they and their parents were willing to participate in the study. Randomization was performed by a staff member who was not involved in the trial. Afterwards, 1834 participants agreed and completed the study, which included physical measurements, questionnaire survey records and biochemical variable tests, with $87 \%$ response rate. Children with missing information such as age ( $n$ 16), sex ( $n$ 20) or BMI ( $n$ 15) were excluded, as were those who had a serious health problem ( $n$ 13), including any physical and psychological conditions that compromised their participation in the study (e.g. disabled children, serious cognitive or psychological dysfunction, congenital heart disease). In addition, to minimize any possible bias related to ethnicity, all participants were required to be ethnic Han. Finally, a total of 1770 children and adolescents ( 857 boys and 913 girls) aged 7-17 years were enrolled in the study.

\section{Data collection and measurements}

Data on children were collected using a standard parent/ guardian questionnaire filled in by one of their parents in a face-to-face interview. Before the survey, all eligible investigators were invited to attend an organized training session, wherein the purpose of the study, administration of the questionnaire, the standard method of measurement, the importance of standardization and the study procedures were explained. A strict test was administered after the training session and only those who obtained a perfect score in the test were accepted as investigators. The internal inspectors provided further instructions and support during data collection. Data regarding demographic characteristics, child birth and feeding situation and parental education level were obtained, as well as family history of chronic diseases, which was defined as the presence of chronic diseases in at least one parent. Chronic diseases included hypertension, diabetes (types 1 and 2) and obesity. Parental education level was classified into three categories: none/primary ( $<6$ years of schooling), secondary (6-12 years) and university or above ( $>12$ years). The completed questionnaires were reviewed by trained staff and uploaded into the database.

All participants underwent a complete anthropometric evaluation to ascertain their nutritional status. The variables assessed were weight, height, waist circumference (WC), hip circumference (HC) and BMI (weight/height ${ }^{2}$, $\mathrm{kg} / \mathrm{m}^{2}$ ). Fasting body weight was measured to the nearest $0 \cdot 1 \mathrm{~kg}$ on a double ruler scale (RGT-140; Wujin Hengqi Co. Ltd, Changzhou, China). Height was measured to an accuracy of $1 \mathrm{~mm}$ with a free-standing stadiometer mounted on a rigid tripod (GMCS-I; Xindong Huateng Sports Equipment Co. Ltd, Beijing, China) by trained interviewers following a standardized procedure. Each participant was asked to stand erect with back, buttocks and heels in continuous contact with the vertical height rod of the stadiometer and head orientated in the Frankfurt plane. The horizontal headpiece was then placed on top of the head of the participant to measure his/her height. WC (Myotape; Jiete Co. Ltd, Dongguan, China) was measured using the horizontal plane midway between the lowest rib and the upper border of the iliac crest at the end of normal inspiration and expiration, whereas HC was measured at the maximum extension of the buttocks. The average of two consecutive measures was used in the analyses. Waist-to-height ratio was calculated as WC (in centimetres) divided by height (in centimetres), whereas waist-to-hip ratio was calculated by dividing WC by HC.

Blood pressure was measured using a mercury sphygmomanometer (model 501010009; Yuyue Medical Co. Ltd, Jiangsu, China) after each participant had rested for at least $15 \mathrm{~min}$ in a sitting position. Diastolic pressure was defined as the point of disappearance of Korotkoff sound (fifth phase). Blood pressure was taken twice on the right arm with an appropriately sized cuff. 
The average of two readings obtained at a minimum of 5 min apart was recorded.

\section{Laboratory tests}

Biochemical variables included fasting glucose (FG), TAG, total cholesterol, HDL-C and LDL cholesterol. After $12 \mathrm{~h}$ of overnight fasting, venous blood samples were taken from the antecubital vein and collected into vacuum tubes. The samples were then centrifuged at $3000 \mathrm{rpm}$ for $10 \mathrm{~min}$ (Avanti $^{\circledR} \mathrm{J}-301$; Beckman Coulter Co. Ltd, Shanghai, China), then serum or plasma was separated and stored at $-80^{\circ} \mathrm{C}$ for subsequent analysis. All biochemical analyses on blood parameters were carried out by a biomedical analysis company, which was accredited by Peking University. The glucose oxidase method was used to measure FG, HDL-C was calculated using the clearance method and total cholesterol and fasting TAG were assayed using enzymatic methods.

\section{Definitions}

MetS was defined mainly according to the criteria of the International Diabetes Federation (IDF; 2007) ${ }^{(16)}$ adapted for children and adolescents as abdominal obesity (defined by WC $\geq 90$ th percentile according to the cut-off points for Chinese children) and the presence of two or more clinical features, including TAG $\geq 1.7 \mathrm{mmol} / \mathrm{l}, \mathrm{HDL}-\mathrm{C}<1.03 \mathrm{mmol} / \mathrm{l}$, systolic blood pressure $\geq 130 \mathrm{mmHg}$ and/or diastolic blood pressure $\geq 85 \mathrm{mmHg}$, and serum $\mathrm{FG} \geq 5.6 \mathrm{mmol} / \mathrm{l}$. LBW and HBW were defined as birth weight $<2500 \mathrm{~g}$ and $\geq 4000 \mathrm{~g}$, respectively, for newborns on term without congenital malformations. Passive smoking was considered present when at least one parent smoked cigarettes.

MetS was also defined in the sensitivity analysis by using other criteria proposed by Cook et al. ${ }^{(17)}$, to be comparable with other publications.

\section{Statistical analysis}

Descriptive statistics were calculated for all of the variables, including continuous variables (reported as mean values and standard deviations) and categorical variables (reported as numbers and percentages). The differences between sexes and ages were evaluated using the Student's $t$ test, ANOVA, non-parametric test or the $\chi^{2}$ test, as appropriate. Correlation coefficients between MetS components and its associated factors were calculated as well. Multivariate logistic regression analyses (Enter method) were employed to identify the independent factors of MetS, and odds ratios and corresponding $95 \%$ confidence intervals were calculated. The multivariate analysis in the second step was sex-adjusted and age-adjusted to control the influence of puberty on the dependent variable. All statistical analyses were performed using the statistical software package SPSS version $16 \cdot 0$, and $P$ values less than $0 \cdot 05 / 0 \cdot 01$ were considered to be statistically significant.

\section{Results}

\section{Characteristics of the study population}

The baseline characteristics of the participants, including anthropometry, MetS features, birth and feeding situations, as well as family environment are shown in Table 1. A total of 1770 interviewed children and adolescents, including 913 girls (51.6\%) and 857 boys (48.4\%) with a mean age of 11.3 (SD 2.9) years, were enrolled in the study. Boys had higher mean values of variables such as height, weight, BMI, waist-to-hip ratio, WC, FG, systolic and diastolic blood pressure than girls. In terms of birth and feeding information, the participants were born weighing 3238.5 (SD 481.6) g on average, and $66.3 \%$ were exclusively breast-fed for $\geq 30 \mathrm{~d}$. With regard to family environments, the parental education levels of the target population were $1.5 \%$ and $2.4 \%, 40 \cdot 4 \%$ and $43.1 \%$, and $54.7 \%$ and $50.4 \%$ for father and mother with none/primary, secondary and university level education or above, respectively. In addition, the percentages of passive smoking and family history of hypertension, diabetes and obesity were $37.2 \%, 52.7 \%, 28.0 \%$ and $24.4 \%$, respectively. No significant differences were observed in family environments between boys and girls.

\section{Prevalence and features of metabolic syndrome}

Among children less than 10 years old, the prevalence of abdominal obesity, hypertension, high FG, low HDL-C and high TAG was $11.2 \%, 0.5 \%, 0.8 \%, 7 \cdot 4 \%$, and $2 \cdot 1 \%$, respectively, which were all lower than the corresponding figure in children older than 10 years. The overall prevalence of MetS in children and adolescents was $1.1 \%$ among children aged 10-17 years (Table 2), which was higher in boys (1.4\%) than in girls $(0.8 \% ; P<0.001)$. Furthermore, boys had a higher rates of high FG (3.2\% v. 0.8\%), low HDL-C (16.4\%v. 11.6\%) and high TAG ( $8.3 \% v .6 .5 \%)$ but lower rates of abdominal obesity (10.3\% v. $14 \cdot 1 \%)$ and hypertension ( $0.9 \% v \cdot 1 \cdot 0 \%)$ than girls. A total of $564(31.1 \%)$ participants had at least one MetS feature, among which 453 (25.4\%), ninety-one (5.1\%) and nineteen ( $1.1 \%)$ participants had one, two and three MetS features, respectively.

Figure 1 shows the prevalence of MetS and metabolic disorders among 7- to 17-year-old children in various age groups in Guangzhou, China. The prevalence of MetS in 10-, 11- and 12-year-old groups was $2.0 \%, 3.6 \%$ and $2.7 \%$, respectively, which were higher than in other age groups. This difference was also observed in the groups with one metabolic disorder and two metabolic disorders.

The MetS presence in the sensitivity analysis was based on the criterion defined by Cook et al. ${ }^{(17)}$. Its prevalence was $2.5 \%, 3.6 \%$ and $1.5 \%$ among all children aged $7-17$ years, boys and girls, respectively (see online supplementary material, Table S1). The prevalence of MetS in 10-, 11- and 12 -year-olds was $3.2 \%, 4.5 \%$ and $3.6 \%$, respectively, which was also higher than in other age groups (see online supplementary material, Fig. S1). 
Table 1 Demographic and clinical characteristics of the 7- to 17-year-old children and adolescents ( $n$ 1770), Guangzhou, China, September 2013

\begin{tabular}{|c|c|c|c|c|c|c|c|}
\hline \multirow[b]{2}{*}{ Variable } & \multicolumn{2}{|c|}{ Total $(n$ 1770) } & \multicolumn{2}{|c|}{ Boys ( $n$ 857) } & \multicolumn{2}{|c|}{ Girls (n 913) } & \multirow[b]{2}{*}{$P$ value } \\
\hline & Mean or $n$ & SD or $\%$ & Mean or $n$ & SD or $\%$ & Mean or $n$ & SD or $\%$ & \\
\hline Age (years)§ & $11 \cdot 3$ & $2 \cdot 9$ & $11 \cdot 3$ & $2 \cdot 9$ & $11 \cdot 3$ & 2.9 & 0.92 \\
\hline \multicolumn{8}{|l|}{ Anthropometry§ } \\
\hline Height $(\mathrm{m})$ & 1.46 & 0.17 & 1.47 & 0.18 & 1.45 & 0.17 & 0.01 \\
\hline Weight (kg) & $40 \cdot 1$ & $15 \cdot 1$ & $42 \cdot 0$ & $15 \cdot 9$ & 38.2 & $14 \cdot 1$ & 0.01 \\
\hline BMI $\left(\mathrm{kg} / \mathrm{m}^{2}\right)$ & $18 \cdot 3$ & 6.5 & $18 \cdot 8$ & 4.6 & $17 \cdot 8$ & 7.9 & 0.01 \\
\hline $\mathrm{HC}(\mathrm{cm})$ & $76 \cdot 5$ & $30 \cdot 8$ & $76 \cdot 1$ & $19 \cdot 8$ & $76 \cdot 8$ & 38.5 & 0.58 \\
\hline WHR & 0.85 & 0.09 & 0.87 & 0.07 & 0.85 & 0.12 & 0.01 \\
\hline WHtR & 0.44 & 0.09 & 0.45 & 0.06 & 0.44 & $0 \cdot 11$ & 0.21 \\
\hline \multicolumn{8}{|l|}{ Metabolic syndrome components§ } \\
\hline WC $(\mathrm{cm})$ & 64.9 & $18 \cdot 7$ & $65 \cdot 6$ & $11 \cdot 7$ & $64 \cdot 2$ & 23.5 & 0.09 \\
\hline $\mathrm{FG}(\mathrm{mmol} / \mathrm{l})$ & 4.6 & 0.5 & 4.7 & 0.5 & 4.5 & 0.4 & 0.01 \\
\hline HDL-C (mmol/l) & 1.4 & 0.3 & 1.4 & 0.3 & 1.4 & 0.3 & 0.21 \\
\hline TAG (mmol/l) & 0.9 & 0.5 & 0.9 & 0.5 & 0.9 & 0.4 & 0.93 \\
\hline $\mathrm{SBP}(\mathrm{mmHg})$ & 98.7 & $22 \cdot 0$ & $100 \cdot 8$ & $17 \cdot 9$ & $96 \cdot 6$ & $25 \cdot 0$ & 0.01 \\
\hline $\mathrm{DBP}(\mathrm{mmHg})$ & $62 \cdot 7$ & $13 \cdot 3$ & $63 \cdot 3$ & $7 \cdot 2$ & $62 \cdot 1$ & $17 \cdot 2$ & 0.05 \\
\hline \multicolumn{8}{|l|}{ Birth weight and breast-feeding } \\
\hline Exclusively breast-fed $\geq 30 \mathrm{~d} \|$ & 1174 & $66 \cdot 3$ & 562 & $65 \cdot 6$ & 612 & $67 \cdot 0$ & 0.85 \\
\hline Breast-feeding duration (months)§ & $6 \cdot 27$ & $2 \cdot 6$ & $6 \cdot 22$ & 2.6 & $6 \cdot 31$ & 2.6 & 0.58 \\
\hline Birth weight $(\mathrm{g}) \S$ & 3238.5 & 481.6 & 3296.5 & $486 \cdot 7$ & $3185 \cdot 6$ & 471.0 & 0.01 \\
\hline Low birth weightll & 63 & 3.6 & 27 & 3.2 & 36 & 3.9 & $0 \cdot 10$ \\
\hline High birth weightll & 86 & 4.9 & 50 & $5 \cdot 8$ & 36 & 3.9 & 0.40 \\
\hline \multicolumn{8}{|l|}{ Family environment\| } \\
\hline Paternal education level & & & & & & & 0.78 \\
\hline None/primary & 26 & 1.5 & 12 & 1.5 & 13 & 1.4 & \\
\hline Secondary & 721 & $40 \cdot 4$ & 354 & $40 \cdot 4$ & 371 & $40 \cdot 5$ & \\
\hline University or above & 976 & 54.7 & 468 & 54.0 & 508 & 55.4 & \\
\hline Maternal education level & & & & & & & 0.34 \\
\hline None/primary & 45 & $2 \cdot 4$ & 24 & $2 \cdot 8$ & 21 & $2 \cdot 3$ & \\
\hline Secondary & 768 & 43.1 & 378 & 43.6 & 390 & $42 \cdot 5$ & \\
\hline University or above & 905 & $50 \cdot 4$ & 428 & $49 \cdot 4$ & 744 & $52 \cdot 0$ & \\
\hline Family history of hypertension & 941 & $52 \cdot 7$ & 441 & $50 \cdot 9$ & 500 & 54.5 & 0.29 \\
\hline Family history of diabetes & 500 & 28.0 & 246 & 28.4 & 254 & $27 \cdot 7$ & 0.33 \\
\hline Family history of obesity & 436 & 24.4 & 222 & $25 \cdot 6$ & 214 & $23 \cdot 3$ & 0.52 \\
\hline Passive smoking & 663 & $37 \cdot 2$ & 316 & 36.5 & 347 & $37 \cdot 8$ & 0.80 \\
\hline
\end{tabular}

HC, hip circumference; WHR, waist-to-hip ratio; WHtR, waist-to-height ratio; WC, waist circumference; FG, fasting glucose; HDL-C, HDL cholesterol; SBP, systolic blood pressure; DBP, diastolic blood pressure.

$\ddagger P$ value between sexes was calculated using the $x^{2}$ test for categorical variables and the two-sample $t$ test for continuous variables.

$\S$ Data are presented as mean and standard deviation.

IIData are presented as number and frequency.

\section{Association between metabolic syndrome features and associated factors}

The multiple logistic regression method was applied to analyse the relationship between MetS and its associated factors. The results in Table 3 show that HBW, not LBW, was significantly associated with abdominal obesity $(\mathrm{OR}=2 \cdot 86 ; 95 \%$ CI $1.62,5.06)$ and MetS (OR=3.61; $95 \%$ CI $1.33,9.82)$ even after adjustment for sex and age. In addition, $>6$ months of maternal breast-feeding was inversely correlated with the risk of MetS $(\mathrm{OR}=0.39$; $95 \%$ CI $0 \cdot 16,0 \cdot 98)$.

\section{Discussion}

A series of currently available studies indicates that MetS is not only present in adults but also in children and adolescents. However, the early-life factors for MetS in children and adolescents are uncertain. The present study, which included
Chinese children and adolescents, showed that the overall prevalence of MetS among the participants was $1.1 \%$, in which higher prevalence was observed in boys than in girls. Most importantly, HBW was significantly associated with abdominal obesity and MetS. Furthermore, breast-feeding for longer than 6 months in children and adolescents during their early life was inversely associated with MetS in later life. To the best of our knowledge, the current study is the first study exploring the relationship between MetS using the IDF criteria and early-life factors among children aged 7-17 years living in South China.

MetS was identified in nineteen $(1.1 \%)$ children and adolescents in the sample population. The prevalence varied depending on the criteria applied because no universally accepted definition of childhood MetS is available. The main criteria of MetS adopted in the present study were proposed by the IDF in 2007, and these criteria have been used worldwide for comparison of data from different countries $^{(16)}$. In 2009, Zorzi et al. reported that the 
Table 2 Prevalence of metabolic syndrome and its components in 7- to 17-year-old children and adolescents ( $n$ 1770), Guangzhou, China, September 2013

\begin{tabular}{|c|c|c|c|c|c|c|c|}
\hline \multirow[b]{2}{*}{ Characteristic } & \multicolumn{2}{|c|}{ Total (n 1770) } & \multicolumn{2}{|c|}{ Boys ( $n$ 857) } & \multicolumn{2}{|c|}{ Girls (n 913) } & \multirow[b]{2}{*}{$P$ value $\ddagger$} \\
\hline & $n$ & $\%$ & $n$ & $\%$ & $n$ & $\%$ & \\
\hline \multicolumn{8}{|l|}{$<10$ years old } \\
\hline Number & 618 & 34.9 & 302 & $35 \cdot 2$ & 316 & 34.6 & \\
\hline Abdominal obesity & 69 & $11 \cdot 2$ & 41 & $13 \cdot 6$ & 28 & 8.9 & 0.041 \\
\hline Hypertension & 3 & 0.5 & 3 & 1.0 & 0 & - & - \\
\hline High FG & 5 & 0.8 & 5 & 1.7 & 0 & - & - \\
\hline Low HDL-C & 46 & 7.4 & 24 & 7.9 & 22 & $7 \cdot 0$ & 0.377 \\
\hline High TAG & 13 & $2 \cdot 1$ & 8 & $2 \cdot 6$ & 5 & 1.6 & 0.260 \\
\hline \multicolumn{8}{|l|}{$\geq 10$ years old } \\
\hline Number & 1152 & $65 \cdot 1$ & 555 & $64 \cdot 8$ & 597 & 65.4 & \\
\hline Abdominal obesity & 141 & $12 \cdot 3$ & 57 & $10 \cdot 3$ & 84 & $14 \cdot 1$ & 0.030 \\
\hline Hypertension & 11 & 1.0 & 5 & 0.9 & 6 & 1.0 & 0.550 \\
\hline High FG & 23 & $2 \cdot 0$ & 18 & $3 \cdot 2$ & 5 & 0.8 & 0.003 \\
\hline Low HDL-C & 160 & 13.9 & 91 & $16 \cdot 4$ & 69 & 11.6 & 0.010 \\
\hline High TAG & 85 & 7.4 & 46 & $8 \cdot 3$ & 39 & $6 \cdot 5$ & 0.152 \\
\hline \multicolumn{8}{|c|}{ Pattern of risk factor clustering } \\
\hline 0 component & 1322 & 74.8 & 638 & 74.5 & 684 & $75 \cdot 0$ & 0.432 \\
\hline 1 component & 453 & $25 \cdot 4$ & 224 & $25 \cdot 9$ & 229 & $25 \cdot 0$ & 0.70 \\
\hline 2 components & 91 & $5 \cdot 1$ & 58 & $6 \cdot 7$ & 33 & 3.6 & 0.01 \\
\hline$\geq 3$ components & 19 & $1 \cdot 1$ & 12 & 1.4 & 7 & $0 \cdot 8$ & 0.25 \\
\hline Metabolic syndrome & 19 & $1 \cdot 1$ & 12 & 1.4 & 7 & 0.8 & 0.25 \\
\hline
\end{tabular}

FG, fasting glucose; HDL-C, HDL cholesterol.

$\ddagger P$ value between sexes was calculated using the $x^{2}$ test for categorical variables.

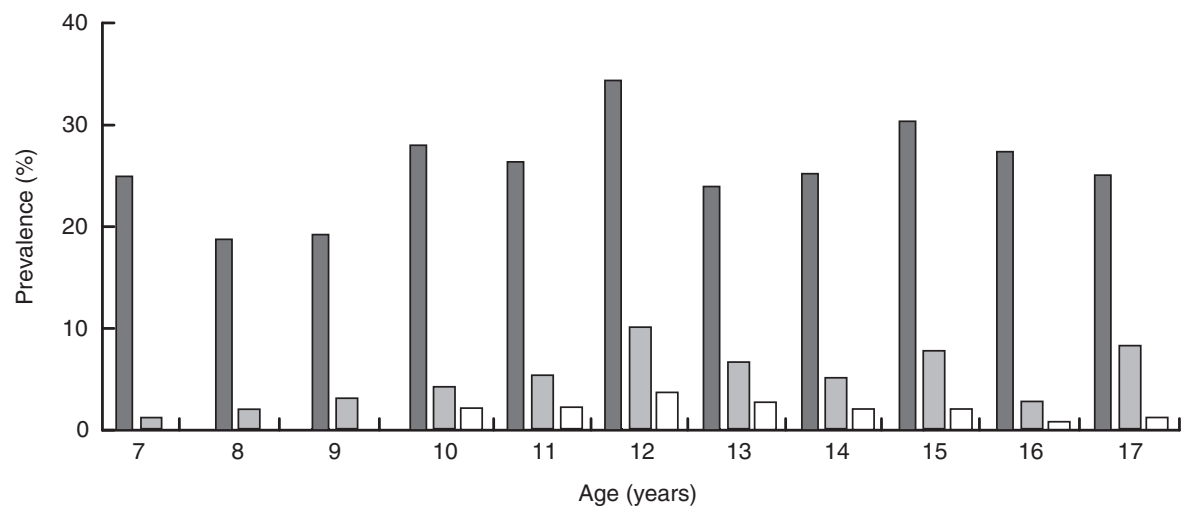

Fig. 1 Prevalence of the metabolic syndrome $(\square)$ and of one metabolic disorder $(\square)$ and two metabolic disorders ( $\square$ ) among 7- to 17 -year-old children and adolescents $(n$ 1770) in various age groups in Guangzhou, China, September 2013

prevalence of MetS in Canadian Tsimshian Nation youth aged $6-18$ years was $4.7 \%$ using the IDF criteria $^{(18)}$. In 2009, Cizmecioglu et al. reported that the prevalence of MetS among 2491 Turkish schoolchildren aged 10-19 years was $2 \cdot 3 \%$ according to IDF guidelines ${ }^{(19)}$. The results obtained in the present study indicated a lower prevalence of MetS in the current study than in Western countries but slightly higher than that in another study conducted in China, in which the overall prevalence of MetS for children older than 10 years was $0 \cdot 8 \%$, in accordance with the IDF definition as well ${ }^{(3)}$. However, the estimated prevalence of MetS was much higher $(2.5 \%)$ when the definitions proposed by Cook et al. were applied. Applying the IDF criteria for children aged 10 years or above may result in underestimation of the real situation, because MetS diagnosis was not suggested by the IDF definition for children below 10 years old. The sensitivity of the IDF definition in MetS diagnosis was significantly lower than the sensitivity of Cook et al.'s definition. In addition, the IDF definition considers central obesity as an essential element. Therefore, the lower prevalence of MetS in the present study than in Western countries may be partially explained by the lower prevalence of obesity among Chinese children.

An age difference in the prevalence of MetS components was found in the present study, which is consistent with previous studies. A study of children and adolescents from Iran and Germany found that young Iranians and Germans aged 6-10 years had a lower risk of MetS than those aged $10-16$ years $(1.0 \% v .2 \cdot 0 \%$ for Iranians, $0.1 \% v \cdot 0.5 \%$ for Germans) ${ }^{(20)}$. The potential for early onset of puberty in 
adolescents may potentially increase the risk for MetS ${ }^{(21)}$ Changes in the hormone regulation of appetite, satiety and fat distribution during puberty may place adolescents at increased risk for additional weight gain.

Abdominal obesity and low HDL-C level are the most prevalent features among the abnormalities in the current study. In other countries, abdominal obesity was also found to be the most prevalent among the five MetS components in adolescents. By using the IDF definition, the overall prevalence of abdominal obesity in adolescents was $34.7 \%$ and $8.4 \%$ in Americans and Koreans, respectively ${ }^{(22)}$. Therefore, obese adolescents with abdominal obesity should be an important target for intervention. In addition, using the IDF criteria in the United Arab Emirates ${ }^{(23)}$, a country having one of the highest rates of diabetes in the world, the low HDL-C prevalence $(88 \%$ and $74 \%$ in boys and girls, respectively) was extremely higher than that observed in the present study, whereas approximately $11 \%$ prevalence rate was observed in southern Italians ${ }^{(24)}$. Another study conducted in Brazil, Iran and Germany indicated that genetics might be a crucial reason for the above results ${ }^{(20)}$. Nevertheless, these differences could be due to the use of different methodologies (e.g. the clearance method was used for HDL-C in the current study, but the phosphotungstic acid precipitation method was used for Caucasian children).

Identification of early-life risk factors is essential for developing early interventions to prevent MetS. HBW (i.e. not LBW) was found to be directly related to the development of abdominal obesity and MetS in the present study. A previous report indicated a J-shaped relationship between birth weight and fat mass later in life, with a higher prevalence of obesity seen in individuals with $\mathrm{HBW}^{(25)}$. The association between $\mathrm{LBW}$ or $\mathrm{HBW}$ and MetS is controversial, with some studies showing a strong relationship ${ }^{(25,26)}$ and others not ${ }^{(27)}$. A study conducted in Spain showed that birth weight and positive MetS diagnosis are associated in both sexes ${ }^{(28)}$. By contrast, Khuc et al. ${ }^{(29)}$ surveyed 357 adolescents in Chile and did not find any association between birth weight and early development of MetS. The mechanism is still not completely understood, but it may involve either altered appetite regulation or insulin secretion ${ }^{(30)}$, and even hypothalamic inflammation ${ }^{(31)}$. Moreover, maternal overnutrition is deleterious to the health of the offspring and could result in a phenotype that is a characteristic of Met $S^{(32)}$.

For maternal breast-feeding, the results showed an inverse association between participants who had been breast-fed for $>6$ months and positive MetS diagnosis in childhood and adolescence; this inverse association is consistent with those obtained in other studies with similar populations of children and adolescents ${ }^{(33)}$. Ekelund et $a l^{(34)}$ emphasized that participants who had been breast-fed for more than 6 months benefited from maternal breast-feeding in terms of MetS prevention. Breast-feeding offers some protection related to the development of obesity ${ }^{(35)}$. Considering that breast-fed infants gain weight more slowly over the first year compared with 
formula-fed infants ${ }^{(36)}$, infant weight gain may pertain to the mechanism that decreases obesity risk in those who were breast-fed. Breast-feeding is also associated with lower risk of hypercholesterolaemia, hypertension, diabetes, glucose intolerance and insulin resistance ${ }^{(37,38)}$. More importantly, the significant effects of breast-feeding were independent, suggesting that the effect of breast-feeding on MetS may not be mediated by weight gain during infancy. The association between breast-feeding and abdominal obesity was not found in the present study; this aspect needs further exploration.

Childhood socio-economic status affects the risk of cardiometabolic diseases. However, the relationship between childhood socio-economic status and MetS remains inconclusive, one reason being the insufficiency of data from Asian countries. No association between the prevalence of MetS and parental education was found in the current study, which is consistent with another study conducted in a national representative sample of Chinese adolescents $^{(39)}$. However, a study conducted in Koreans found independent and positive associations between maternal education and occupational status and MetS ${ }^{(13)}$. Many factors contribute to the development of MetS, including physical activity level, dietary intake and socio-economic status, some of which are related to one another, and this correlation may be insignificantly associated with MetS.

The present study adds to the current knowledge about child birth weight and breast-feeding and their long-term health effects, which might have important implications for the management of pregnancies and child care. Given the increase in obesity prevalence among children and adults, these findings have implications for the cycle of obesity and its consequences like MetS in subsequent generations. We encourage primary-care providers to discuss the consequences of breast-feeding as part of anticipatory guidance early in the care of children.

Several limitations of the study should be mentioned. First, causation could not be inferred because the study applied a cross-sectional analysis. The specific roles of maternal breast-feeding and birth weight in MetS development could not be determined with certainty. Second, as indicated by the IDF definition, children younger than 10 years old cannot be diagnosed with MetS and the adolescents in our study included different stages of puberty, with labile adiposity. Therefore, the results among younger children in our study should be interpreted with caution. Third, breast-feeding data were retrospectively obtained from the parents. Therefore, the breast-feeding data were possibly overestimated or underestimated. The study also has many strengths, such as the high quality control of physical examinations and the good response rate of participants. In addition, although several studies have reported an association between birth weight and individual components of MetS, no previous study in China had focused on birth weight, breast-feeding duration and MetS components as potential risk factors.

\section{Conclusion}

In conclusion, the present results showed the prevalence of MetS among children and adolescents. The sensitivity of the IDF definition in diagnosing MetS was significantly lower when applied in China. Therefore, an appropriate diagnostic tool is needed to identify MetS in Chinese children. Moreover, HBW was significantly associated with abdominal obesity and MetS, and breast-feeding children for longer than 6 months was inversely associated with MetS.

\section{Acknowledgements}

Acknowledgements: The authors thank all the participants, as well as the doctors and nurses involved in the study for anthropometric measurement and data collection. Financial support: This work was supported by Guangdong Science and Technology Program (grant number 2014A020220002) and special research grant for non-profit public service of the Ministry of Health of China (grant number 201202010), which had no role in the design, analysis or writing of this article. Conflict of interest: The authors declare no conflict of interest. Authorship: Y.Z. is the co-first author and J.J. is the co-corresponding author of this study. Y.C., J.J. and Y.Z. designed the experiments; J.M. carried out the experiments; J.W. analysed data and wrote the draft of the manuscript; all authors were involved in writing the paper and had final approval of the submitted and published versions. Ethics of buman subject participation: Written informed consent was obtained from adolescents and their parents. This study was approved by the Ethics Committee of the School of Public Health, Sun Yat-Sen University.

\section{Supplementary material}

To view supplementary material for this article, please visit http://dx.doi.org/10.1017/S1368980015002542

\section{References}

1. Grundy SM, Brewer HJ, Cleeman JI et al. (2004) Definition of metabolic syndrome: report of the National Heart, Lung, and Blood Institute/American Heart Association conference on scientific issues related to definition. Circulation 109, 433-438.

2. Miller JM, Kaylor MB, Johannsson M et al. (2014) Prevalence of metabolic syndrome and individual criterion in US adolescents: 2001-2010 National Health and Nutrition Examination Survey. Metab Syndr Relat Disord 12, 527-532.

3. Xu H, Xu G, Ma J et al. (2012) Prevalence of the metabolic syndrome among children from six cities of China. BMC Public Health 12, 13.

4. Morrison JA, Friedman LA, Wang P et al. (2008) Metabolic syndrome in childhood predicts adult metabolic syndrome and type 2 diabetes mellitus 25 to 30 years later. $J$ Pediatr 152, 201-206. 
5. Ahmadi A, Gharipour M, Nouri F et al. (2013) Metabolic syndrome in Iranian youths: a population-based study on junior and high schools students in rural and urban areas. J Diabetes Res 2013, 738485.

6. Eckel RH, Grundy SM \& Zimmet PZ (2005) The metabolic syndrome. Lancet 365, 1415-1428.

7. Zhu S, St-Onge MP, Heshka S et al. (2004) Lifestyle behaviors associated with lower risk of having the metabolic syndrome. Metabolism 53, 1503-1511.

8. Burke V, Beilin LJ, Simmer K et al. (2005) Predictors of body mass index and associations with cardiovascular risk factors in Australian children: a prospective cohort study. Int J Obes (Lond) 29, 15-23.

9. Alberti G, Zimmet P, Shaw J et al. (2004) Type 2 diabetes in the young: the evolving epidemic: the international diabetes federation consensus workshop. Diabetes Care 27, 1798-1811.

10. Singh R, Shaw J \& Zimmet P (2004) Epidemiology of childhood type 2 diabetes in the developing world. Pediatr Diabetes 5, 154-168.

11. Li C, Johnson MS \& Goran MI (2001) Effects of low birth weight on insulin resistance syndrome in Caucasian and African-American children. Diabetes Care 24, 2035-2042.

12. Wang X, Liang L, Junfen FU et al. (2007) Metabolic syndrome in obese children born large for gestational age. Indian J Pediatr 74, 561-565.

13. Choi BY, Lee DC, Chun EH et al. (2014) The relationship between metabolic syndrome and childhood maternal education level, job status: findings from the Korean National Health and Nutrition Examination, 2007-2009. Korean J Fam Med 35, 207-215.

14. Rodriguez-Moran M \& Guerrero-Romero F (2006) Hyperinsulinemia in healthy children and adolescents with a positive family history for type 2 diabetes. Pediatrics 118 e1516-e1522.

15. Yu S, Guo X, Yang H et al. (2014) An update on the prevalence of metabolic syndrome and its associated factors in rural northeast China. BMC Public Health 14, 877.

16. Zimmet P, Alberti KG, Kaufman F et al. (2007) The metabolic syndrome in children and adolescents - an IDF consensus report. Pediatr Diabetes 8, 299-306.

17. Cook S, Weitzman M, Auinger P et al. (2003) Prevalence of a metabolic syndrome phenotype in adolescents: findings from the third National Health and Nutrition Examination Survey, 1988-1994. Arch Pediatr Adolesc Med 157, 821-827.

18. Zorzi A, Wahi G, Macnab AJ et al. (2009) Prevalence of impaired glucose tolerance and the components of metabolic syndrome in Canadian Tsimshian Nation youth. Can J Rural Med 14, 61-67.

19. Cizmecioglu FM, Etiler N, Hamzaoglu O et al. (2009) Prevalence of metabolic syndrome in schoolchildren and adolescents in Turkey: a population-based study. J Pediatr Endocrinol Metab 22, 703-714.

20. Schwandt P, Kelishadi R, Ribeiro RQ et al. (2010) A threecountry study on the components of the metabolic syndrome in youths: the BIG Study. Int J Pediatr Obes 5, 334-341.

21. Jasik CB \& Lustig RH (2008) Adolescent obesity and puberty: the 'perfect storm'. Ann N Y Acad Sci 1135, 265-279.

22. Park J, Hilmers DC, Mendoza JA et al. (2010) Prevalence of metabolic syndrome and obesity in adolescents aged 12 to 19 years: comparison between the United States and Korea. J Korean Med Sci 25, 75-82.
23. Mehairi AE, Khouri AA, Naqbi MM et al. (2013) Metabolic syndrome among Emirati adolescents: a school-based study. PLoS One 8, e56159.

24. Noto D, Niglio T, Cefalu AB et al. (2009) Obesity and the metabolic syndrome in a student cohort from southern Italy. Nutr Metab Cardiovasc Dis 19, 620-625.

25. Dubois L \& Girard M (2006) Early determinants of overweight at 4.5 years in a population-based longitudinal study. Int J Obes (Lond) 30, 610-617.

26. Mi J, Law C, Zhang KL et al. (2000) Effects of infant birthweight and maternal body mass index in pregnancy on components of the insulin resistance syndrome in China. Ann Intern Med 132, 253-260.

27. Hirschler V, Bugna J, Roque M et al. (2008) Does low birth weight predict obesity/overweight and metabolic syndrome in elementary school children? Arch Med Res 39, 796-802.

28. Gonzalez-Jimenez E, Montero-Alonso MA, Schmidt-RioValle J et al. (2014) Metabolic syndrome in Spanish adolescents and its association with birth weight, breastfeeding duration, maternal smoking, and maternal obesity: a cross-sectional study. Eur J Nutr 54, 589-597.

29. Khuc K, Blanco E, Burrows R et al. (2012) Adolescent metabolic syndrome risk is increased with higher infancy weight gain and decreased with longer breast feeding. Int J Pediatr 2012, 478610.

30. Portha B, Chavey A \& Movassat J (2011) Early-life origins of type 2 diabetes: fetal programming of the $\beta$-cell mass. Exp Diabetes Res 2011, 105076.

31. Rother E, Kuschewski R, Alcazar MA et al. (2012) Hypothalamic JNK1 and IKK $\beta$ activation and impaired early postnatal glucose metabolism after maternal perinatal high-fat feeding. Endocrinology 153, 770-781.

32. Armitage JA, Taylor PD \& Poston L (2005) Experimental models of developmental programming: consequences of exposure to an energy rich diet during development. J Physiol 565, 3-8.

33. Yang Z \& Huffman SL (2013) Nutrition in pregnancy and early childhood and associations with obesity in developing countries. Matern Child Nutr 9, Suppl. 1, 105-119.

34. Ekelund U, Anderssen S, Andersen LB et al. (2009) Prevalence and correlates of the metabolic syndrome in a population-based sample of European youth. Am J Clin Nutr 89, 90-96.

35. von Kries R, Koletzko B, Sauerwald T et al. (1999) Breast feeding and obesity: cross sectional study. BMJ $\mathbf{3 1 9}$, $147-150$

36. Dewey KG, Heinig MJ, Nommsen LA et al. (1992) Growth of breast-fed and formula-fed infants from 0 to 18 months: the DARLING Study. Pediatrics 89, 1035-1041.

37. de Armas MG, Megias SM, Modino SC et al. (2009) Importance of breastfeeding in the prevalence of metabolic syndrome and degree of childhood obesity. Endocrinol Nutr 56, 400-403.

38. Vaag A (2009) Low birth weight and early weight gain in the metabolic syndrome: consequences for infant nutrition. Int $J$ Gynaecol Obstet 104, Suppl. 1, S32-S34.

39. Li Y, Yang $\mathrm{X}$, Zhai $\mathrm{F}$ et al. (2008) Prevalence of the metabolic syndrome in Chinese adolescents. Br J Nutr $\mathbf{9 9}$, 565-570. 